Substances marked with an asterisk were separated by chemical methods analogous to those used by Szilard and Chalmers ${ }^{1}$. The radioactive manganese was separated from a solution of potassium permanganate, the radioactive $\mathrm{P}^{32}$ from carbon disulphide.

We tried to relate our results to Fermi's theory of B-ray decay. For this purpose it is necessary to calculate for each radioactive element Fermi's ${ }^{2}$ function $F\left(\eta_{0}\right)$, where $\eta_{0}=H_{p \max .} / 1700$. According to Fermi, the values of $\tau . F\left(\eta_{0}\right)$ should be distributed in two groups, differing from each other by $a$ factor of about 100. The first group should correspond to allowed transitions, the second to the forbidden ones. As can be seen from the table, such a sharp separation into two distinct groups, as obtained by Fermi for natural $\beta$-active elements, can scarcely be found for the substances we investigated; also, the data obtained by us do not fit the well-known Sargent diagram.$$
\text { Physical-Technical Institute, }
$$$$
\text { A. I. Altchanow. }
$$$$
\text { A. I. Alichanian. }
$$$$
\text { B. S. Dželepow. }
$$

Leningrad. July 3.

1. L. Szilard and T. A. Chalmers, NATURE, 134, $462 ; 1934$. B. Fermi, Z. Phys., B, 88, 161; 1934 .

\section{Velocity of 'Slow Neutrons'}

A NEUTRON source (beryllium and 300 millicurie radon) was placed in the centre of a block of paraffin wax, of about $20 \mathrm{~cm}$. diameter in all dimensions. After emerging from the paraffin, the neutrons had to pass, on their way to the detecting apparatus, through the marginal parts of a wooden disc of $50 \mathrm{~cm}$. diameter and $2 \mathrm{~cm}$. thickness, which could be made to rotate with a speed of 4,000 rev. per minute. This speed corresponds to a velocity of about $9 \times 10^{3}$ $\mathrm{cm}$./sec. of the parts of the wood through which the neutrons penetrated. From former experience one would expect each slow neutron, while passing through the dise, to suffer a number of collisions with the protons contained in the wood, and therefore the velocity of the disc would be superimposed on their velocity distribution with the disc at rest. Assuming that a large part of the neutrons have thermal velocities, which are for neutrons of the order of $2 \times 10^{\mathrm{s}} \mathrm{cm} . / \mathrm{sec}$, an asymmetrical distribution of the directions of the neutrons emerging from the rotating disc would result, about ten per cent more slow neutrons being emitted in the direction of rotation than in the opposite direction. ${ }^{1}$

In order to get evidence of this asymmetry, we placed a cadmium screen with a hole of $6 \mathrm{~cm} . \times 6 \mathrm{~cm}$. near the rotating disc, defining thereby a region where the neutrons were allowed to emerge from the wood. Two boron-lined ionisation chambers were placed behind the screen in such a way that they collected the neutrons projected at less than $45^{\circ}$ and $135^{\circ}$ relative to the direction of motion, respectively. The chambers could in turn be connected to a linear amplifier and mechanical counter ; and alternative countings of equal duration were made with the disc rotating alternately one way or the other.

The experiments revealed indeed an asymmetry of the expected order of magnitude. Adding together the counts of the chamber which lay in the direction of motion of the disc, we get 11,100 particles against
10,644 for the corresponding sum of the counts in the other chamber. The observed difference is, therefore, $4 \cdot 2$ per cent, with a probable error of one per cent. When comparing this value with the beforementioned theoretical value of 10 per cent, we must take into account that only part of the counts was really due to the action of slow neutrons. By screening off the slow neutrons by means of a cadmium plate, the number of counts was reduced to about one half; these remaining counts were probably partly due to fast neutrons, partly to the natural contamination of the chamber. The result of the experiment may, therefore, be taken as evidence that the main part of those neutrons which are strongly absorbed in cadmium have very nearly thermal velocities.

In conclusion, we would like to thank Prof. J. Franck for many stimulating discussions.

\section{O. R. Frisch.}

E. T. SørEnsEn.

Institute for Theoretical Physics, Copenhagen. 1 Experiments with a similar arrangement, but yet on slightly
different lines, have recently been performed by Amaldi, d'Agostino, Fermi, Pontecorvo and Segrè, La Ricerca Scientifica, vi, 1, No. 11-12.

\section{Activity of Androstendione on the Sexual Organs of the Male Rat}

It has repeatedly been stated that the relation between the results of capon test and rat test varies with preparations having male hormone action ${ }^{1,2}$. A definite number of capon units, applied in the form of testicular extracts, has a stronger activity on the sexual organs of the male rat than pure androsterone or hormone fractions from male urine. Gallagher and Koch ${ }^{2}$, moreover, showed that boiling alkali destroys the hormone action of testicular extracts only, but not of the urine extracts. In fact, androsterone and trans-dehydro-androsterone, the existence of which in the urine has been described by Butenandt, are not altered by treatment with alkali.

Recently, L. Ruzicka and A. Wettstein have prepared trans-dehydro-androsterone from cholesterol and transformed it by oxidation into androstendione of melting point $173^{\circ}-174^{\circ}$. Like corpus luteum hormone, it is an unsaturated diketone, and has the following formula:

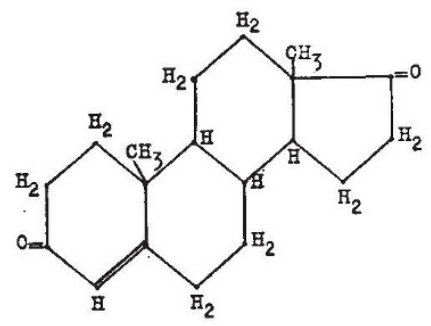

It is now well known that the boiling of corpus luteum extracts with alkali destroys the hormone action. L. Ruzicka and A. Wettstein therefore recently suggested that androstendione or analogous compounds might be responsible for the peculiar action of the testicular extracts. ${ }^{3}$

As Ruzicka and Wettstein have already reported, the capon unit for androstendione and likewise for the saturated androstandione amounts to about $100 \gamma$, according to the 6-day test used in this 